\title{
Intracellular phase for an extracellular bacterial pathogen: MgtC shows the way
}

\author{
Audrey Bernut ${ }^{1, \#}$, Claudine Belon ${ }^{1}$, Chantal Soscia ${ }^{2}$, Sophie Bleves ${ }^{2}$, Anne-Béatrice Blanc-Potard ${ }^{1, *}$ \\ ${ }^{1}$ Laboratoire de Dynamique des Interactions Membranaires Normales et Pathologiques, Université de Montpellier (DIMNP CNRS- \\ UMR5235), Place Eugène Bataillon, 34095 Montpellier Cedex 05, France. \\ ${ }^{2}$ CNRS \& Aix-Marseille University, Laboratoire d'Ingénierie des Systèmes Macromoléculaires (UMR7255), IMM, 31 Chemin Joseph \\ Aiguier, 13402 Marseille cedex 20, France. \\ \# Present address: Centre d'études d'agents Pathogènes et Biotechnologies pour la Santé (CPBS - FRE 3689), 1919 route de Mende, \\ 34293 Montpellier, France. \\ * Corresponding Author: Anne-Béatrice Blanc-Potard, E-mail: anne.blanc-potard@univ-montp2.fr
}

\begin{abstract}
Pseudomonas aeruginosa is an extracellular pathogen known to impair host phagocytic functions. However, our recent results identify MgtC as a novel actor in $P$. aeruginosa virulence, which plays a role in an intramacrophage phase of this pathogen. In agreement with its intracellular function, $P$. aeruginosa $\mathrm{mgtC}$ gene expression is strongly induced when the bacteria reside within macrophages. MgtC was previously known as a horizontally-acquired virulence factor important for multiplication inside macrophages in several intracellular bacterial pathogens. MgtC thus provides a singular example of a virulence determinant that subverts macrophages both in intracellular and extracellular pathogens. Moreover, we demonstrate that $P$. aeruginosa MgtC is required for optimal growth in $\mathrm{Mg}^{2+}$ deprived medium, a property shared by MgtC factors from intracellular pathogens and, under $\mathrm{Mg}^{2+}$ limitation, $P$. aeruginosa MgtC prevents biofilm formation. We propose that MgtC has a similar function in intracellular and extracellular pathogens, which contributes to macrophage resistance and fine-tune adaptation to the host in relation to the different bacterial lifestyles. MgtC thus appears as an attractive target for antivirulence strategies and our work provides a natural peptide as MgtC antagonist, which paves the way for the development of MgtC inhibitors.
\end{abstract}

There is growing evidence that pathogens recognized as extracellular can actually survive inside macrophages after phagocytosis to hide from the immune system and spread, but little is known about the intracellular behavior of these pathogens and about the bacterial factors involved. For example, Staphylococcus aureus has long been considered as an extracellular pathogen, but infections in the zebrafish embryo model have contributed to reveal an intracellular phase in phagocytic cells in S. aureus life cycle. P. aerugino$s a$ is known to impair host phagocytic functions, mainly by using cytotoxic and anti-phagocytic determinants. Unexpectedly, our recent work revealed a novel bacterial factor implicated in a macrophage intracellular stage during $P$. aeruginosa acute infection.

Common strategies can be used by bacterial pathogens that share similar lifestyle or similar environmental niches. MgtC is a virulence factor common to several intracellular pathogens including Salmonella enterica serovar Typhimurium (S. Typhimurium) and Mycobacterium tuberculosis. Importantly, MgtC has been shown to promote intramacrophage survival of Yersinia pestis, which is a facultative intracellular pathogen that replicates mainly extracellularly and produces anti-phagocytic factors. In Salmonella, MgtC promotes pathogenicity by inhibiting the bacterial $\mathrm{F}_{1} \mathrm{~F}_{\mathrm{o}}$ ATP synthase. Salmonella mgtC is regulated both at the transcriptional and post-transcriptional levels, with a positive regulation by $\mathrm{Mg}^{2+}$ deprivation and increase in cytosolic ATP, and a negative regulation by the MgtR peptide. In agreement with the $\mathrm{Mg}^{2+}$ regulation, $\mathrm{MgtC}$ has been involved in adaptation to low $\mathrm{Mg}^{2+}$ environments.

The environmental bacterium and opportunistic human pathogen $P$. aeruginosa is a major cause of mortality in cystic fibrosis (CF) patients. Interestingly, $\mathrm{mgtC}$ has been highlighted as a horizontally-acquired gene shared by several opportunistic bacteria infecting CF patients, including P. aeruginosa, Burkholderia ceanocepacia and Mycobacte-

MICROREVIEW on: Belon C, Soscia C, Bernut A, Laubier A, Bleves S, Blanc-Potard AB. A macrophage subversion factor is shared by intracellular and extracellular pathogens. PLoS Pathogens 2015 11(6): e1004969. doi: 10.1371/journal.ppat.1004969 
rium abscessus. MgtC exhibits a sporadic distribution in Pseudomonas species, being mostly associated with strains that are pathogenic for humans and insects. Our phylogenetic analysis indicated that the MgtC protein from the extracellular pathogen $P$. aeruginosa clustered in the same subgroup as MgtC proteins from intracellular pathogens. We investigated the role of $P$. aeruginosa MgtC by analyzing the infection phenotypes and behavior in $\mathrm{Mg}^{2+}$ deprived conditions of an $\mathrm{mgtC}$ mutant.

To evaluate the role of MgtC in P. aeruginosa virulence, we used the zebrafish (Danio rerio) embryo model, which is a model of choice to investigate the contribution of cells from the innate immune system during infection. This model possesses professional phagocytes that can engulf and kill $P$. aeruginosa upon a systemic infection. We showed that the $P$. aeruginosa mgtC mutant is attenuated for acute infection in zebrafish embryos. MgtC most likely acts by protecting $P$. aeruginosa against phagocytes since macrophage depletion (using pu.1 morpholinos) suppressed the difference between mgtC mutant and wildtype PAO1 strain. This hypothesis was supported by ex vivo experiments since the $\mathrm{mgtC}$ mutant is more phagocytosed and more sensitive to bacterial killing than the wild-type strain in $\mathrm{J774}$ macrophages. Hence, similarly to intracellular pathogens, the MgtC virulence determinant of $P$. aeruginosa plays a role towards macrophages. We propose that $P$. aeruginosa has acquired a macrophage subversion factor to resist killing in case of phagocytosis by macrophages and further studies will be required to explore the fate of intracellular bacteria.

In agreement with the intramacrophage role of $P$. aeruginosa $\mathrm{MgtC}$, expression of the $\mathrm{mgtC}$ gene is highly induced when the bacteria reside inside macrophages. To our knowledge, this is the first report of a $P$. aeruginosa gene induced within phagocytic cells. We further demonstrated that phagosome acidification contributes to an

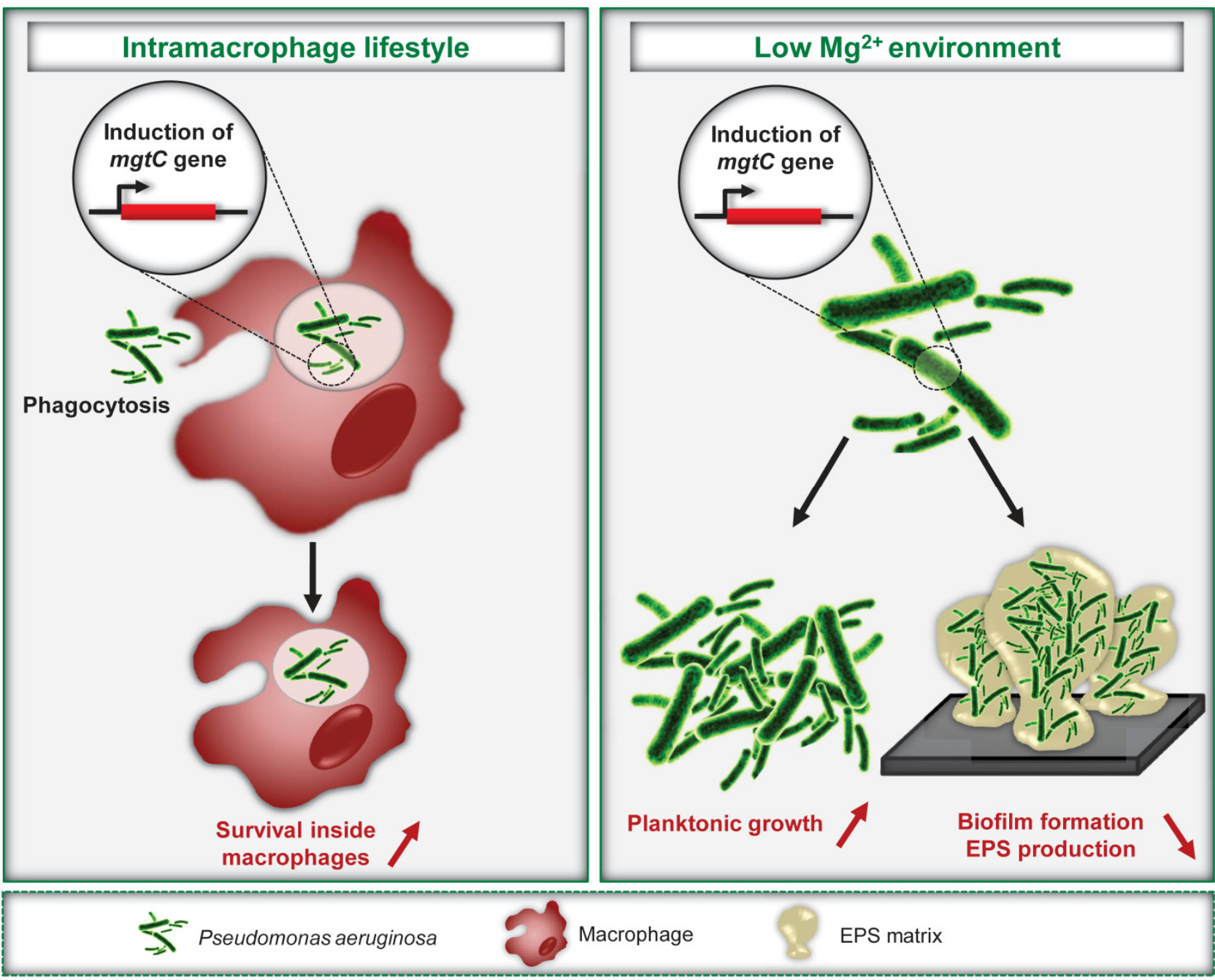

FIGURE 1: $P$. aeruginosa MgtC promotes an intramacrophage phase and limits biofilm formation. Expression of $P$. aeruginosa mgtC gene is induced inside macrophages and in magnesium-deprived medium. $P$. aeruginosa $\mathrm{MgtC}$ is involved in resistance to macrophage, a feature shared with MgtC proteins from intracellular pathogens as $S$. Typhimurium. Under magnesium deprivation, MgtC promotes planktonic growth but limits biofilm formation and EPS production. 
optimal expression of Pseudomonas mgtC. A strong intramacrophage induction has also been reported for the Salmonella $\mathrm{mgtC}$ gene. The intracellular signal that induces Salmonella mgtC transcription is still a matter of debate, but one hypothesis is a combination of cationic peptides and acidic $\mathrm{pH}$. An open task is to uncover the molecular basis for the conservation of $m g t C$ regulation among different species.

We established that $m g t C$ expression in $P$. aeruginosa is induced in conditions of $\mathrm{Mg}^{2+}$ starvation, which is also the case for $m g t C$ genes from intracellular pathogens. Growth kinetic analyses showed that MgtC is required for optimal growth in $\mathrm{Mg}^{2+}$ deprived medium in P. aeruginosa, another common feature of intracellular pathogens. Our results thus indicate a mechanism of convergent evolution between pathogens in their ability to use and regulate MgtC to adapt to $\mathrm{Mg}^{2+}$ limitation. We speculate that this feature does not account for the role of $P$. aeruginosa MgtC in macrophages because addition of extracellular $\mathrm{Mg}^{2+}$ did not rescue the increased sensitivity of $\mathrm{mgtC}$ mutants to macrophages.

$P$. aeruginosa virulence and resistance to treatment is largely due to its ability to form biofilms, whose formation/stability can be affected by extracellular cations, including magnesium ions. Bacterial adherence to glass was visualized and quantified using crystal violet staining to infer the ability of strains to form biofilms. We observed that MgtC expression limits biofilm formation under $\mathrm{Mg}^{2+}$ limiting conditions. The biofilm phenotype of the $\mathrm{mgtC}$ mutant is associated with increased production of exopolysaccharides (EPS), which are essential biofilm matrix components. Interestingly, a recent report of Groisman's laboratory provides an unsuspected link between MgtC, cellulose and intramacrophage survival in Salmonella since cellulose production, which is repressed by MgtC in low $\mathrm{Mg}^{2+}$ medium, was shown to impede Salmonella replication inside macrophages. Cellulose is not produced by $P$. aeruginosa strains, but our finding of an increased EPS production by the $P$. aeruginosa $m g t C$ mutant in low $\mathrm{Mg}^{2+}$ medium, suggests a potential link between EPS production and the role of $P$. aeruginosa $\mathrm{MgtC}$ in macrophages. Further studies will be required to determine the nature of the EPS involved, to investigate their production intracellularly and the putative link to intramacrophage phenotypes.
Our findings highlight the importance of macrophage subversion by $P$. aeruginosa in a model of acute infection. Being a determinant that plays a role during infection both in intracellular and extracellular pathogens, MgtC appears as a promising new target for antivirulence strategies. We have proposed the MgtR membrane peptide (issued from Salmonella) as a natural MgtC antagonist because in $S$. Typhimurium, the intramacrophage replication of a wildtype strain can be reduced upon over-production of MgtR, which plays a negative regulatory role on MgtC expression. $P$. aeruginosa does not encode any MgtR homologue but in a bacterial two-hybrid system, MgtR interacts with both $S$. Typhimurium and $P$. aeruginosa MgtC. We have found that the phenotypes observed with Pseudomonas mgtC mutant in animal and cellular infection models can be mimicked upon heterologous production of the Salmonella MgtR peptide in a wild-type $P$. aeruginosa strain. Our results thus support an action of MgtR peptide as an antagonist of $\mathrm{MgtC}$ and paves the way for the development of antivirulence strategies based on MgtC inhibition.

\section{ACKNOWLEDGMENTS}

This work was supported by Vaincre La Mucoviscidose (IC1002, IC0902 and RF20110600446/1/1/47), the French Ministry of Research (MRT fellowship), the Fondation for Medical Research (FRM FDT20140930905), and Association Gregory Lemarchal (RF20140501165).

\section{CONFLICT OF INTEREST}

The authors declare no competing financial interest.

\section{COPYRIGHT}

(C) 2015 Bernut et al. This is an open-access article released under the terms of the Creative Commons Attribution (CC BY) license, which allows the unrestricted use, distribution, and reproduction in any medium, provided the original author and source are acknowledged.

Please cite this article as: Audrey Bernut, Claudine Belon, Chantal Soscia, Sophie Bleves, Anne-Béatrice Blanc-Potard (2015). Intracellular phase for an extracellular bacterial pathogen: MgtC shows the way. Microbial Cell 2(9): 353-355. doi: 10.15698/mic2015.09.227 